Research Paper

\title{
Tuning of Alternative Splicing - Switch From Proto- Oncogene to Tumor Suppressor
}

\author{
Aleksandra Shchelkunova1, Boris Ermolinsky¹, Meghan Boyle¹, Ivan Mendez¹, Michael Lehker1, Karen S. \\ Martirosyan $^{2}$ and Alexander V. Kazansky ${ }^{1,3}$ \\ 1. Department of Biomedicine, The University of Texas at Brownsville, Brownsville, TX 78520, USA; \\ 2. Department of Physics and Astronomy, The University of Texas at Brownsville, Brownsville, TX 78520, USA; \\ 3. Department of Cellular and Molecular Biology, Baylor College of Medicine, Houston, TX 77030, USA.
}

$\triangle$ Corresponding author: Department of Biomedicine, BRHB 1.122, The University of Texas at Brownsville, 80 Fort Brown, Brownsville, TX 78520, USA. Tel: 1 (956) 882-5780; Email: Alexander.Kazansky@utb.edu.

(C) Ivyspring International Publisher. This is an open-access article distributed under the terms of the Creative Commons License (http://creativecommons.org/ licenses/by-nc-nd/3.0/). Reproduction is permitted for personal, noncommercial use, provided that the article is in whole, unmodified, and properly cited.

Received: 2012.09.09; Accepted: 2012.12.07; Published: 2012.12.19

\begin{abstract}
STAT5B, a specific member of the STAT family, is intimately associated with prostate tumor progression. While the full form of STAT5B is thought to promote tumor progression, a naturally occurring truncated isoform acts as a tumor suppressor. We previously demonstrated that truncated STAT5 is generated by insertion of an alternatively spliced exon and results in the introduction of an early termination codon. Present approaches targeting STAT proteins based on inhibition of functional domains of STAT's, such as DNA-binding, cooperative binding (protein-protein interaction), dimerization and phosphorylation will halt the action of the entire gene, both the proto-oncogenic and tumor suppressor functions of Stat5B.

In this report we develop a new approach aimed at inhibiting the expression of full-length STAT5B (a proto-oncogene) while simultaneously enhancing the expression of STAT5 $\Delta B$ (a tumor suppressor). We have demonstrated the feasibility of using steric-blocking splice-switching oligonucleotides (SSOs) with a complimentary sequence to the targeted exon-intron boundary to enhance alternative intron/exon retention (up to $10 \%$ ). The functional effect of the intron/exon proportional tuning was validated by cell proliferation and clonogenic assays. The new scheme applies specific steric-blocking splice-switching oligonucleotides and opens an opportunity for anti-tumor treatment as well as for the alteration of functional abilities of other STAT proteins.
\end{abstract}

Key words: STAT proteins, RNA splicing, tumor suppressor, splice-switching oligonucleotides, cell-cycle progression.

\section{INTRODUCTION}

The STAT (Signal Transducer and Activator of Transcription) proteins are a family of cytoplasmic transcription factors that were originally identified in the mid-1990's [1, 2]. To date, two predominant STAT isoforms have been identified: a full-length transcription factor and a C-terminal truncated isoform lacking the transactivation domain $[3,4]$. We previously determined that the truncated STAT isoform is gener- ated by retention of an alternatively spliced intron (Figure 1), resulting in the insertion of an early termination codon [4]. The resultant STAT5 $\Delta$ protein, which lacks a transactivation domain, is then expressed. Phosphorylated STAT5 $\Delta$ can translocate to the nucleus and bind to specific promoter sequences, thereby blocking the transcription of downstream genes due to lack of the transactivation domain. These 
truncated isoforms have been identified for STATs 1, 3,4 , STAT $5 \mathrm{a}$ and $5 \mathrm{~b}$, and have been shown to be the product of alternative splicing [4-6]. While at least one group showed that STAT isoforms are a result of post-transcriptional proteolytic cleavage, it was noted that this event does not occur in vivo [6].

Constitutive activation of STAT5 proteins has been demonstrated in many diverse human cancer cell lines and clinical tumors including those of the prostate [7-14]. A clear understanding of STAT5 function in vivo has been greatly hindered by the existence of two nearly identical genes, STAT5A and STAT5B, which share $93 \%$ homology at the amino acid level. An additional complication stems from the existence of naturally occurring C-terminal truncated dominant-negative isoforms of STAT5 [4].

Previous reports have suggested potentially diverse functions for STAT5 isoforms. Although, recent studies provide clear evidence that STAT5B, contributes to tumor progression in epithelial cancers $[11,13$,
$15,16]$, especially those of the prostate [11]. Specific activation of full-length STAT5B in epithelial cells representing invasive and metastatic prostate cancer has been previously demonstrated $[11,13]$. This finding is consistent with STAT5 being highly activated in high-grade human prostate cancers [9]. Increased activation of STAT5 was also associated with increasingly aggressive behavior of prostate cancer $[9,17]$. In contrast, the naturally occurring dominant-negative truncated isoform STAT $5 \Delta$ B can block cell cycle progression and inhibit growth, invasive potential and clonogenic ability (hallmark of transformed and malignant potential [18]) of cancer cell lines [11, 17, 19, 20]. Furthermore, we demonstrated that STAT $5 \Delta B$ could inhibit the growth of cancer cells in grafting studies in vivo [10, 11]. Thus, the previous research demonstrated role of dominant-negative form of STAT5B as tumor suppressor [11, 17, 19, 20], which blocks cell-cycle progression.

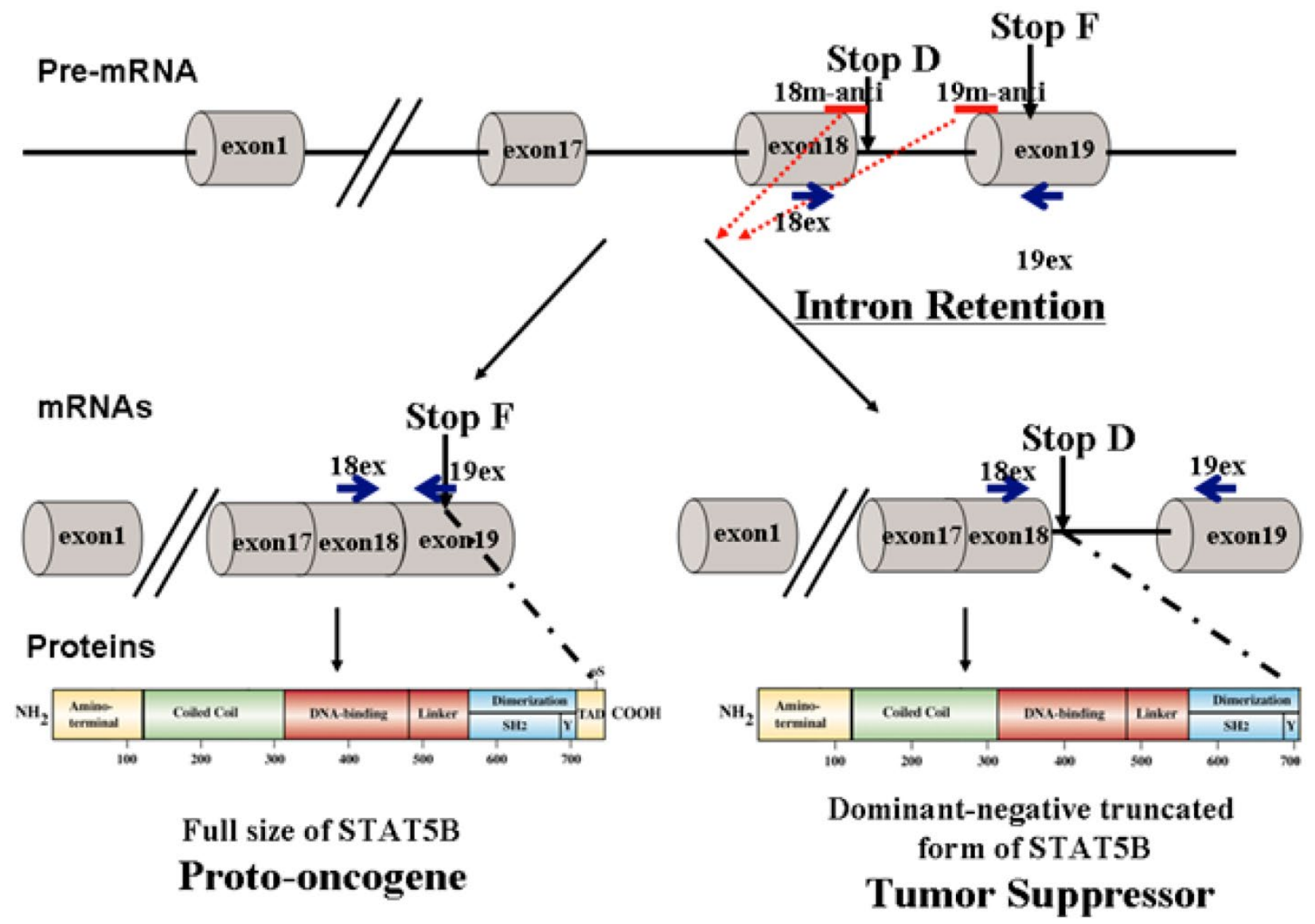

Figure I. Modulation of STAT5B isoforms by antisense oligonucleotides. $18 \mathrm{~m}$-anti and 19 -anti bars above the transcripts indicate antisense splice-switching oligonucleotides. Blue arrows represent oligonucleotides for semi-quantitative RT-PCR analysis of splice-blocking. 
Current therapeutic strategies against STAT5B and other STAT proteins are predominantly based on inhibiting STAT functional domains such as DNA binding, cooperative binding (protein-protein interactions), and dimerization/phosphorylation domains. Inhibition of these key domains not only halts proto-oncogenic functions, but also inhibits tumor suppressor actions of dominant-negative STAT5 $\Delta \mathrm{B}$, therefore diminishing the effectiveness of the therapy. To overcome therapeutic challenges, we developed a novel strategy based on endogenous mechanisms regulating STAT5B isoforms that utilizes a pro-drug capable of converting full length STAT5B (proto-oncogenic) into the dominant negative truncated form of STATB (tumor suppressive). Therefore we applied specific steric-blocking splice-switching oligonucleotides (SSOs) with a complimentary sequence to the targeted exon-intron boundary in the pre-mRNA of Stat5B to induce alternative intron/exon retention enhancing production of tumor suppressor protein. The SSOs complementary to exon/intron boundary sequences can block access of the spliceosomal machinery and stimulate retention of the associated exon [21-23]. They were used to alter splicing for therapeutic purposes in cell line models of human disease [21]. Splice-blocking oligonucleotides should bind specifically to target sequence while at the same time contain extra- or intra-molecular complementarity, have suitable solubility, and do not bind too tightly (favoring off-target effects) or too loosely (causing low efficacy). The SSOs must be stable in serum, hybridize at a high melting temperature, and form duplexes with the targeted RNA, which are not processed by RNase $\mathrm{H}$ and/or other nucleases. This is usually achieved by $2^{\prime}$ modification of the sugar with $O$-methyl, $O$-methoxyethyl or $O$-aminopropyl groups or by backbone modifications such as in $\mathrm{N}^{\prime} \rightarrow \mathrm{P}^{\prime}$ phosphoroamidate, peptide nucleic acids (PNAs), phosphorodiamidate morpholino (PMO), or locked nucleic acids (LNAs) [24-26]. PNAs offer specificity similar to Morpholinos, but their low water solubility makes PNA experiments difficult. Morpholino oligos are usually soluble at several millimolar aqueous concentrations. We have chosen to use Morpholino oligonucleotides, which form stable base pairs with complementary nucleic acid sequences but do not bind proteins to a significant extent, and they mediate RNA cleavage by RNase H or the RISC (RNA induced silencing complex).

In this work, we also developed quantitative PCR methodology to detect splice variants of STAT5B, providing a feasible solution to the significant challenge of separating nearly identical truncated forms of STAT5A and STAT5B. Here, we report evidence of the feasibility of implementing steric-blocking splice-switching morpholino oligonucleotides for the modulation of STAT5B isoforms.

\section{METHODS}

\section{Tissue Culture}

Human PC-3 prostate cancer cells were obtained from American Type Culture Collection (Manassas, VA) and maintained in RPMI 1640 supplemented with $10 \%$ fetal bovine serum.

\section{Morpholino oligonucleotides and transfection}

Morpholino antisense oligonucleotides (m-anti) were purchased from Gene Tools, LLC (Philomath, OR, USA). They were delivered into PC-3 prostate cancer cells using Endo-Porter reagent (Gene Tools, LLC) as per manufacturer protocol.

\section{cDNA production}

Total RNA was isolated $24 \mathrm{hr}$ post-treatment and reverse transcribed using an RNeasy Mini Kit (QIAGEN, CA) and SuperScript ${ } I I I R T$ Reverse Transcriptase (Invitrogen, CA) according to recommended manufacturer protocols.

\section{Sequence and analysis of DNA fragment.}

Sequence of RT-PCR fragments has been performing in both directions by SeqWright DNA technology Services (Houston, TX). Alignment of sequences has been done by BLASTN 2.2.27 against NCBI nucleotide collection.

\section{TagMan probes for SPLICE and NONSPLICE variants of Stat5B}

Real-time PCR was performed via StepOne Real-Time PCR System (ABI) utilizing self-designed TaqMan hydrolysis probes (SPLICE and NONSPLICE) and TaqMan-based ABI gene expression assays (Hs99999905_m1, Gapdh). All qPCR experiments were performed in duplicate. Briefly, cDNA samples were introduced to a MicroAmp Optical 48-well reaction plate (ABI), which was covered with MicroAmp 48-well Optical Adhesive Film (ABI). Each experimental well contained $20 \mu$ total reaction volume and included $10 \mu \mathrm{l}$ of TaqMan Universal PCR Master Mix (ABI), $1 \mu \mathrm{l}$ of 20x TaqMan assay, $8 \mu \mathrm{l}$ of water and $1 \mu \mathrm{l}$ of analyzed sample. Self-designed $20 \mathrm{x}$ assays (SPLICE and NONSPLICE) contained $16 \mu \mathrm{M}$ each of forward and reverse primers and $5 \mu \mathrm{M}$ of TaqMan probe. The final PCR conditions were as follows: pre-heating at $50{ }^{\circ} \mathrm{C}$ for $2 \mathrm{~min}$, denaturation at $95^{\circ} \mathrm{C}$ for $10 \mathrm{~min}$ and 40 cycles of amplification and quantification ( $15 \mathrm{sec}$ at $95^{\circ} \mathrm{C}$ and $60 \mathrm{sec}$ at $60{ }^{\circ} \mathrm{C}$ ). Samples were normalized using Taqman based ABI 
gene expression assay Hs99999905_m1 for human Glyceraldehyde 3-phosphate dehydrogenase (Gapdh, amplicon size $=122 \mathrm{bp}$ ).

Sequence information for designing SPLICE and NONSPLICE splice variant primers and probes was obtained from NCBI NG_007271.1. For this project, SPLICE is considered a transcript variant without introns between exons 18 and 19 while NONSPLICE indicates the presence of the intron between exons 18 and 19. TaqManTM probes, labeled at the $5^{\prime}$ end with FAM (6-carboxyfluorescein) and at the $3^{\prime}$ end with TAMRA (6-carboxytetramethylrhodamine), were synthesized via IDT. Primers and probes for TaqMan hydrolysis qPCR assays specific for each splice variant were designed using the Primer Express v3.0 program (ABI). For SPLICE (Figure 1), the forward (5'CAGCGCCACGTACATGGAC - $\left.3^{\prime}\right)$ and reverse $\left(5^{\prime}\right.$ CGAAGTCCCCATCGGTGTC -3') primer sequences amplify a 75 bp region and probe (5'-FAMAGGACTGAGTCAGGGTTCTGTGGGTACATGTTA TAGT-TAMRA-3') were designed to span the junctions between exons 18-19. For NONSPLICE (Figure 1), the forward (5'- GGCTTTCCCCTTCCCTTCA -3') and reverse (5'- GAAGTCCCCATCGGTGTCAA - $3^{\prime}$ ) primer sequences amplify a $75 \mathrm{bp}$ region, and probe (5'-FAM- CTTTCCCTCCCAAGCCCTGACTCAGTC -TAMRA-3') was designed to span intron-19 exon junctions. Primers and probes are specific to the input template as no other targets were found in selected NCBI Transcript Reference Sequences database. Duplex formation and secondary nucleic acid structures of primer sequences were also evaluated using OligoAnalyzer 3.0 (Integrated DNA Technologies). The analysis of primers was performed by Primer-Blast, encompassed all homo sapien DNA sequences in the NCBI database, and showed only one specific product (http://blast.ncbi.nlm.nih.gov/).

To assess the efficiency of assays and assure validity of the $\triangle \triangle C T$ method, standard curves were prepared for serial dilutions of input cDNA for SPLICE, NONSPLICE and Gapdh (ABI, Hs 999999016_m1) assays. All serial dilutions of human cDNA were prepared and run in triplicate. From the amplification curves, CT (Cq) values for each concentration were determined and the slope of $\mathrm{CT}$ versus nanograms of cDNA was used to calculate the efficiency of amplification (using StepOne software, ABI). TaqMan $^{\mathrm{TM}}$ assays were linear at 6 serial dilutions of cDNA (from 1 to $40 \mathrm{ng}$ ) and the efficiencies and correlation coefficients $\left(R^{2}\right)$ were determined by the average of 2 independent experiments. For SPLICE, the slope was $-3.16, R^{2}=0.997$, efficiency $=107.255$, and for NONSPLICE slope was $-3.223, \mathrm{R}^{2}=0.989$, and efficiency $=104.305$. For the gapdh assay (ABI, Hs 99999905_m1) the slope $=-3.246, \mathrm{R}^{2}=0.989$, efficiency $=103.256$.

In a separate validation experiment to confirm the validity of the $\triangle \triangle C T$ method, standard curves were analyzed for serial dilutions of input cDNA for SPLICE, NONSPLICE and Gapdh. The dilution series of cDNA containing the target gene of interest were analyzed by qPCR. The slopes of the dilution series were calculated by determining $\Delta \mathrm{CT}$ between each point on the dilution series plotted against the log of sample (cDNA) input (mass). The rationale is that in performing $\triangle \triangle C T$ analysis, no or very minimal difference in efficiencies should exist between the gene of interest and the endogenous control (i.e. Gapdh) [27]. For each of these assays, the efficiency and $R^{2}$ values were appropriate and the slope of the line for $\triangle \mathrm{CT}$ of Gapdh and targets (SPLICE and NONSPLICE) was less than 0.1. For qPCR analysis, each sample was run in triplicate. Each run included a control lacking template to test for contamination in assay reagents. After a $94{ }^{\circ} \mathrm{C}$ denaturation for $10 \mathrm{~min}$, reactions were cycled 40 times with a $94{ }^{\circ} \mathrm{C}$ denaturation for $15 \mathrm{sec}$, and a $60{ }^{\circ} \mathrm{C}$ annealing for $1 \mathrm{~min}$. Three types of controls aimed at detecting genomic DNA contamination in the RNA sample or during the RT or qPCR reactions were included: a RT mixture without reverse transcriptase, a RT mixture including the enzyme but no RNA, and a negative control (reaction mixture without cDNA template). The data were collected and analyzed using OneStep Software (ABI).

\section{Preparation of Whole Cell Extracts}

Cells were rinsed twice with ice-cold PBS (Invitrogen, Grand Island, NY), collected via cell scraper, and suspended in radioimmunoprecipitation assay buffer [ $50 \mathrm{mM} \mathrm{NaF}, 10 \mathrm{mM} \mathrm{Na} \mathrm{P}_{2} \mathrm{O}_{7}, 0.5 \%$ sodium deoxycholate, $0.1 \%$ SDS, 1\% NP40, $150 \mathrm{mM} \mathrm{NaCl}, 9.1$ $\mathrm{mM} \mathrm{Na}_{2} \mathrm{HPO}_{4}$, and $1.7 \mathrm{mM} \mathrm{NaH} \mathrm{PO}_{4}$ (pH 7.4)] containing the following protease and phosphatase inhibitors: $1 \mathrm{mM} \mathrm{Na} \mathrm{VO}_{4} ; 1 \mathrm{mM}$ phenylmethylsulfonyl fluoride; $2 \mu \mathrm{g} / \mathrm{ml}$ aprotinin; $2 \mu \mathrm{g} / \mathrm{ml}$ antipain; 2 $\mu \mathrm{g} / \mathrm{ml}$ leupeptin; and $2 \mu \mathrm{g} / \mathrm{ml}$ benzamidine. After incubation at $4{ }^{\circ} \mathrm{C}$ for $30 \mathrm{~min}$, cell extracts were isolated by centrifugation and supernatants were collected. Protein concentrations were measured using the Bio-Rad protein assay reagent (Bio-Rad, Hercules, CA).

\section{Western Blot Analysis}

Protein samples were separated by $7.5 \%$ SDS-PAGE and transferred overnight to an Odyssey Nitrocellulose Membrane (LI-COR Biosciences). We routinely analyzed $60 \mu \mathrm{g}$ of total cell lysate/lane. An antibody recognizing the amino terminus of STAT5 
(N-20) was purchased from Santa Cruz Biotechnology (Santa Cruz, CA, USA), and B-actin (AC-74) antibody was obtained from Sigma (St. Louis, MO, USA). After incubation with primary antibodies, the membranes were washed with PBS containing $0.1 \%$ Tween 20 (PBST) thrice. Membranes were further incubated for $1 \mathrm{hr}$ with IRDye800CW-conjugated goat anti-rabbit IgG and IRDye680-conjugated goat anti-mouse IgG secondary antibodies (LI-COR Biosciences) diluted in Odyssey Blocking Buffer. The blots were then washed three times with PBS-T and rinsed with PBS. Proteins were visualized by scanning the membrane using Odyssey Infrared Imaging System (LI-COR Biosciences) for both 700- and 800-nm wavelengths.

\section{Cell Proliferation Assay}

Cell proliferation assays were performed using the colorimetric 3-(4,5-dimethylthiazol-2-yl)-5-(3carboxymeth-

oxy-phenyl)-2-(4-sulfonyl)-2H-tetrazolium assay (CellTiter 96 AQ; Promega, Madison, WI) according to the manufacturer's protocol. Cells were plated in 96-well plates at a density of $2 \times 10^{3}$ cells/well. After transfection with SSOs, cells were grown for $12 \mathrm{hr}$ in complete media followed by $36 \mathrm{hr}$ in serum-free media.

\section{Clonogenic Assay}

$24 \mathrm{hr}$ after SSOs transfection in six-well plates, PC-3 cells were counted and re-plated in $100 \mathrm{~mm}$ dishes (200 cells/dish) for 10 days in complete medium. Dishes were washed once with $0.85 \mathrm{M} \mathrm{NaCl}$, stained with crystal violet (Sigma, St. Louis, MO, USA) dissolved in ethanol for $15 \mathrm{~min}$, and washed well to remove excess crystal violet. Colonies containing $>50$ cells were scored as positive. Each experiment was performed twice in triplicates.

\section{Statistical Analysis}

Values were expressed as the mean \pm SE. Statistical analyses were performed by Student's $t$ test for paired comparisons using StatView program (Abacus Concepts, Inc., Berkeley, CA, USA). Differences were considered statistically significant at $P<0.05$ and all experiments were performed independently twice or thrice.

\section{RESULTS}

Specific intron retention in Stat5B pre-mRNA by morpholino oligonucleotides in PC-3 cells
PC-3 cells
were treated
with
2'-O-methyl-modified oligoribonucleoside phos- phorothioate with complimentary sequence to the targeted $5^{\prime}$ exon-intron boundary (18m-anti) and to

the $3^{\prime}$ intron-exon boundary (19m-anti) in order to shift splicing patterns as shown in Figure 1 at concentration of $6 \mathrm{uM}$ each. The sequences of $18 \mathrm{~m}$-anti and 19m-anti oligos are TGCCACCTAC/ TTCTGTGGGT and GAGTCAGGG/CTTGGGAGGG AAAGAA, respectively. Underlined is the $9 \mathrm{bp}$ sequence of the $5^{\prime}$ splice site, the slash indicates the exon-intron boundary, and conserved nucleotides of splicing consensus are highlighted in bold. Scrambled mispared oligonucleotide (m-mis) RyrCACrryCTrCTyrGyyr was used as a negative control; where lower-case letters indicate mismatches, $\mathrm{y}$ - pyrimidine nucleotide; and $\mathrm{r}$ - purine nucleotide. Antisense sequences with mismatches were appropriately distributed along the sequence to provide a stringent and realistic assessment of sequence specificity.

Figure 2 shows the transfection of PC-3 cells with steric-blocking splice-switching oligonucleotides. An induced shift in splicing of Stat5b isoforms is present (Figure 2). All targeted Morpholino oligonucleotides generated measurable amounts of predictable product at $559 \mathrm{bp}$ confirmed by sequence analysis. These results demonstrate that the designed oligos are capable of enhancing intron retention. Furthermore, simultaneous introduction of both $18 \mathrm{~m}$-anti and $19 \mathrm{~m}$-anti oligos resulted in an increase of unspliced RT-PCR products (Figure 2, lane 4), suggesting that targeting splice-junctions blocked splicing events. Additionally, we observed that at high concentrations, even non-specific oligonucleotides (m-mis) cause a decrease in cell viability. RNA was isolated $24 \mathrm{hr}$ post-treatment following reverse transcription. To validate the targeting procedure, the level and nature of transcript alteration was characterized by reverse transcriptase (RT)-PCR analysis of splice modification (18ex and 19ex, Figure 1).

To validate the targeting procedure at lower concentration of SSO treatment, we designed a protocol utilizing Quantitative Reverse Transcriptase (RT) PCR analysis of splice modification using custom made TaqMan probes to detect splice variants of STAT5B (refer to Materials and Methods). This procedure detects measurable dose-dependent shift in splicing leading to the retention of specific introns. Next, we treated PC-3 cells with increased concentrations of an equimolar mix of $18 \mathrm{~m}$-anti and 19-anti. Analysis of treated cells was performed on the total RNA isolated $24 \mathrm{hr}$ post-treatment. Figure 3 shows the results of quantitative examination of total RNA by RT-PCR. Results demonstrate tuning of the ratio of non-spliced and spliced forms produced post-incubation with morpholino oligonucleotides. The quantity of STAT5B isoform expression and proportion of non-spliced vs. spliced forms increased 
after treatment by morpholino oligonucleotides. We observed a dose-dependent increase in splicing retention as indicated by a shift in the ratios of the corre- sponding mRNA's while mismatched oligo (m-mis) had no effect on splicing (see Figure 3).

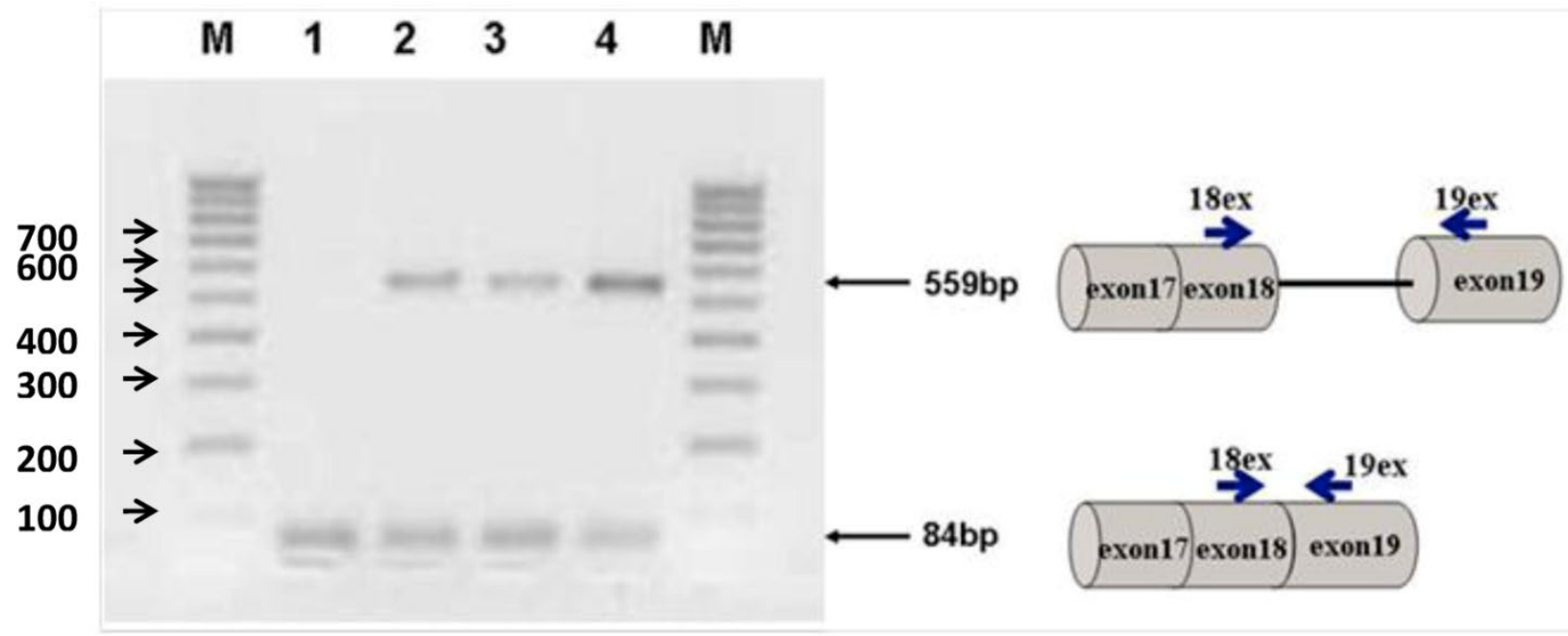

Figure 2. Agarose gel electrophoresis of semi-quantitative RT-PCR splice-blocking products. Splice-junctions were targeted with two splice-blocking Morpholino oligonucleotides as described. Lane I: m-mis; 2: 18m-anti; 3: 19m-anti; 4: combination of I8m-anti and 19m-anti. M - 100 bp ladder. All targeted Morpholino oligonucleotides generated measurable amounts of predictable products indicating splice retention effect. (559bp).

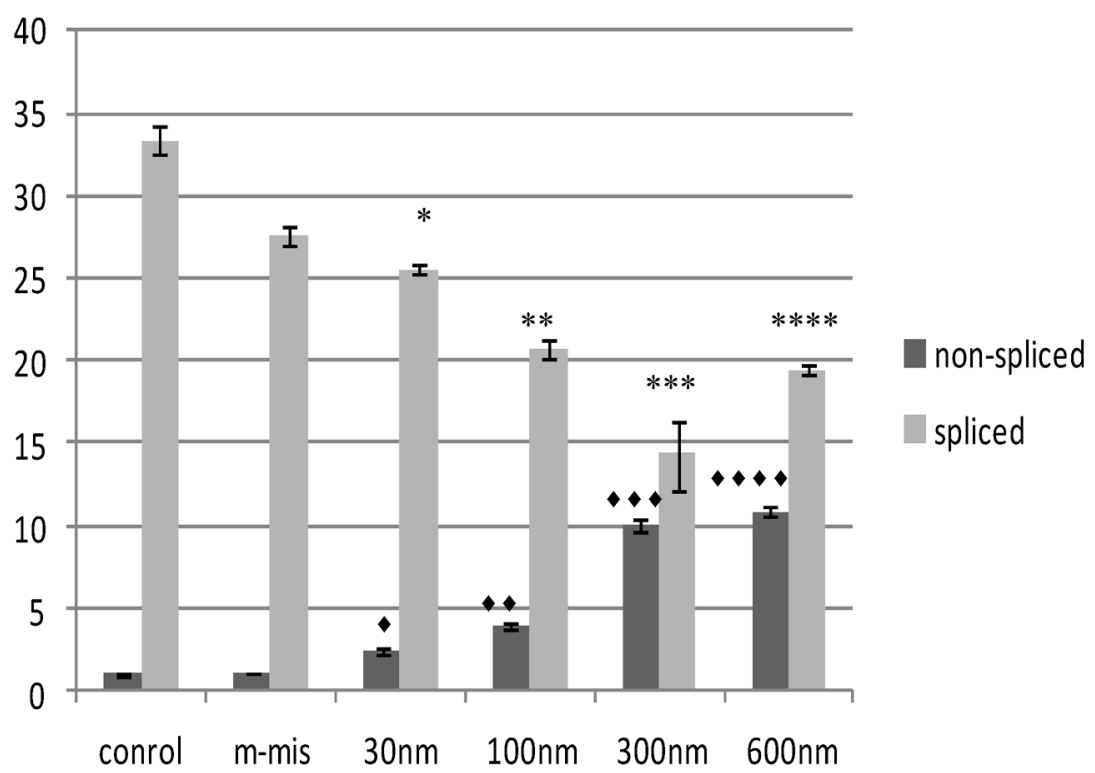

Figure 3. Effect of SSO's treatment on quantity of transcription of Stat5B isoforms. The results of the analysis of total RNA by quantitative RT-PCR. Data demonstrate tuning of the ratio of non-spliced and spliced forms produced after treatment with morpholino oligonucleotides. Control (untreated) cells, m-mis- treatment with $600 \mathrm{nM}$ mispared oligonucleotide, $30 \mathrm{nM}, 100 \mathrm{nM}, 300 \mathrm{nM}, 600 \mathrm{nM}$ treatment with corresponding concentration of the mix of $18 \mathrm{~m}$-anti and 19m-anti. Error bars indicate mean \pm SD $(n=3)$. $\bullet, P=0.002 ; \bullet \bullet$, $P=0.0008 ; \diamond \bullet, P=0.008 ; \diamond \diamond, P=0.0014 ; *, P=0.0016 ; * *, P=0.003 ; * * *, P=0.008 ; * * * *, P=0.0003$. 


\section{Splice regulation by the SSOs induce produc- tion of truncated dominant-negative isoform of STAT5 protein.}

To analyze functional ability of splice blocking effects on protein expression, we performed Western blot analysis. Total protein levels from steric-blocking splice-switching oligonucleotide treated cells revealed an SSO-dependent increase in STAT $5 \Delta \mathrm{B}$ protein production (Figure 4). These data are consistent with RT-PCR results and confirm that splice retention produces a functional transcript that can be efficiently translated into corresponding STAT5 $\Delta \mathrm{B}$ protein.

\section{Inhibition of PC-3 cells proliferation and via- bility by shifting STAT5B isoform toward truncated dominant-negative isoform.}

Previously, we reported that expression of a dominant-negative form of STAT5B reduces cell proliferation and survival by blocking cell cycle progression $[10,11]$. Our aim in manipulating splice switch- ing to create the dominant-negative truncated form from the full form of STAT5B is to produce a potential tumor suppressor. We next analyzed effects of induced splice switching by SSOs on cell proliferation and survival. We have observed dose dependent decrease of cell proliferation rate by SSOs treatment. Quantitative analysis of cell growth was determined by the application of different concentrations of SSOs to tumor cells (Figure 5) and indicated that splice-blocking oligonucleotides decrease the rate of cell proliferation. Colony formation assays (a hallmark of transformed and malignant cell potential [28]) revealed concentration dependent reduction of PC-3 cell viability following SSOs treatment (Figure 6). To distinguish the toxicity of oligos to the cells from STAT5 $\Delta$ B-mediated cell growth retardation, we treated cells with a mismatch morpholino oligonucleotide similar to SSOs but which not cause splice retention.

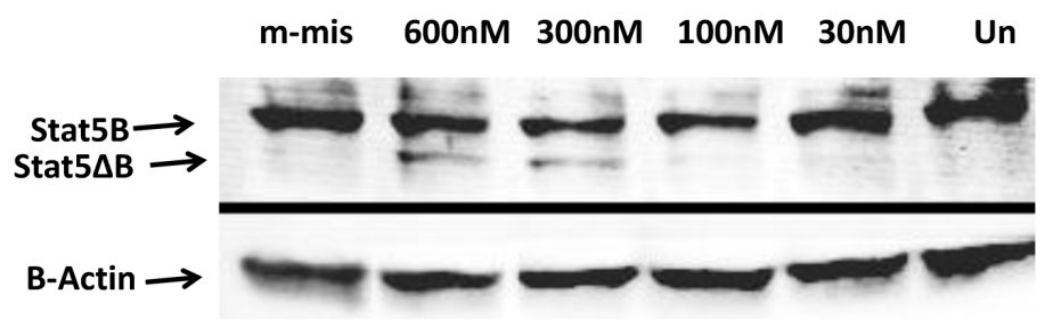

Figure 4. Western Blot analyses of the splice blocking affect of morpholino oligonucleotides. Splice-junctions were targeted with two splice-blocking morpholino oligonucleotides as described above. m-mis - $600 \mathrm{nM}$ of mis-pared oligonucleotide; $600 \mathrm{nM} 300 \mathrm{nM}$, $100 \mathrm{nM}$, and $30 \mathrm{nM}$ corresponding concentration of combination of I8m-anti and 19m-anti; Un -untreated cells.

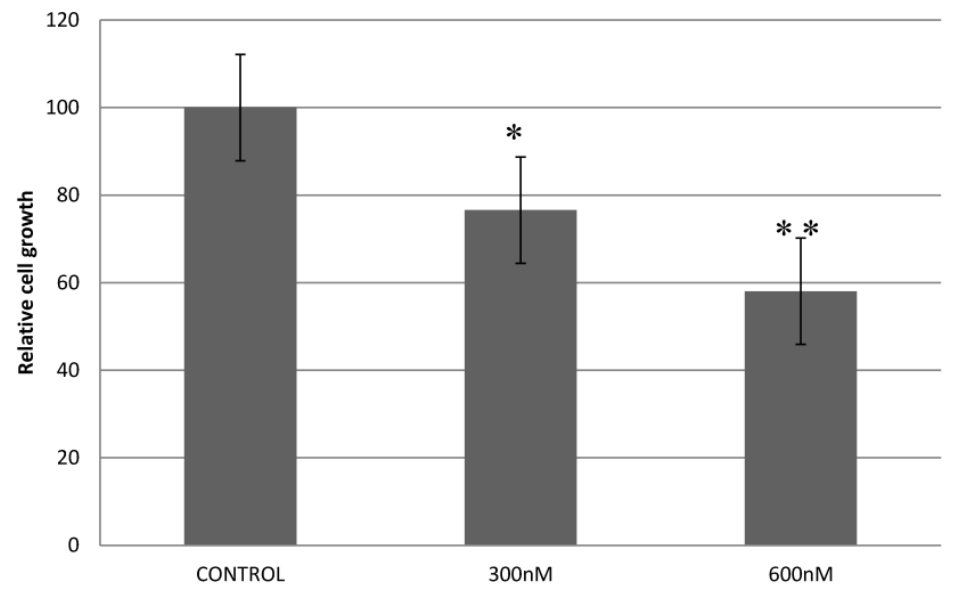

Figure 5. Treatment with splice-blocking oligonucleotides decreases cell proliferation. Control $-600 \mathrm{nM}$ of mispared oligonucleotide; $600 \mathrm{nM}, 300 \mathrm{nM}$ corresponding concentration of combination of $18 \mathrm{~m}$-anti and $19 \mathrm{~m}$-anti. Error bars indicate mean $\pm \mathrm{SD}$ $(\mathrm{n}=3) . *, P=0.009 ; * *, P=0.0115$. 

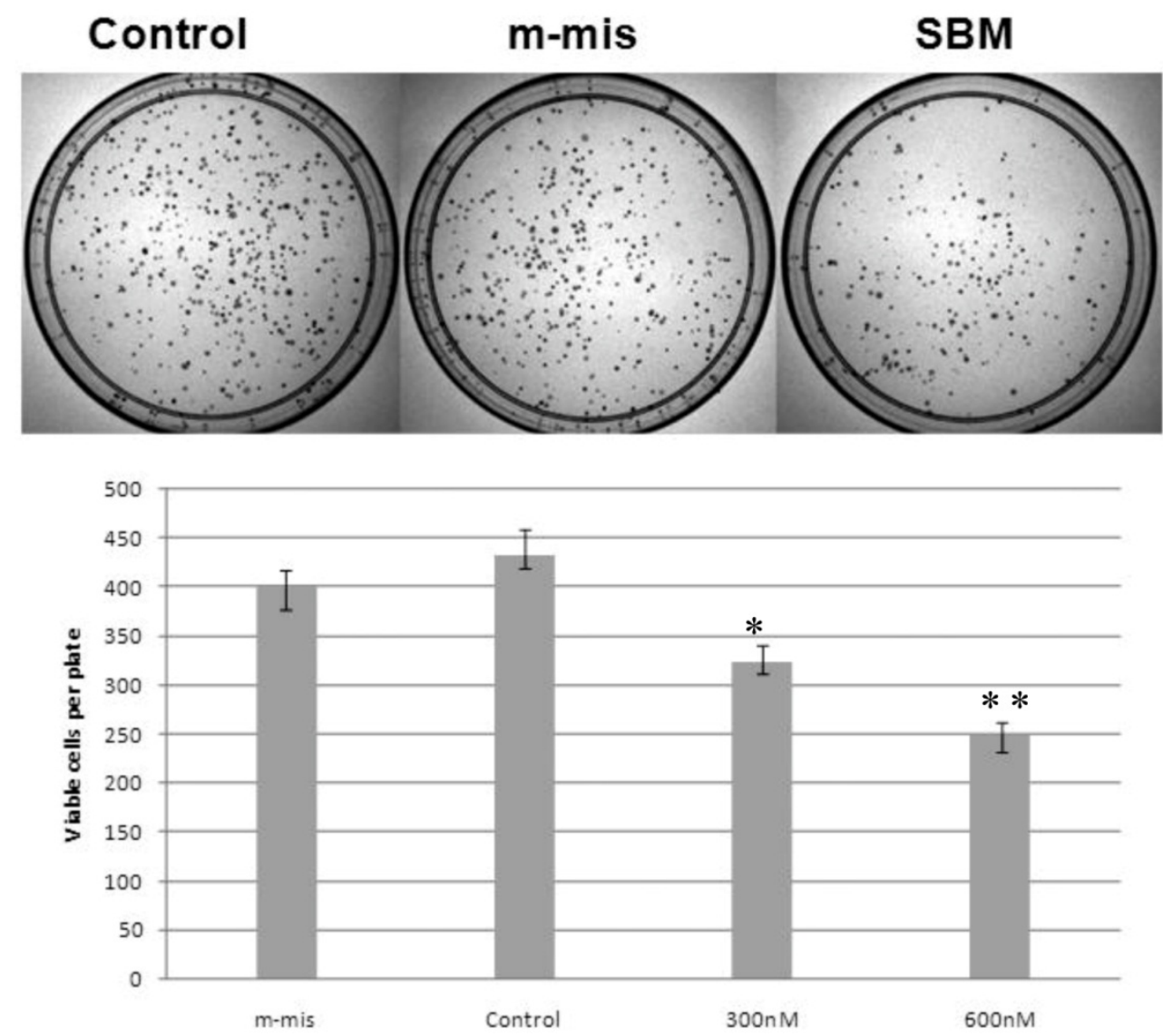

Figure 6. Reduction of cell survival by splice-blocking morpholino-oligonucleotides. Effect of splice blocking on cell viability as determined via clonogenic assay. Top panel shows images of representative plates treated with $600 \mathrm{nM}$ morpholino oligonucleotides while the bottom panel quantitatively represents cell viability of SBM treated cells compared with untreated cells. M-mis - scrambled mispared oligonucleotide was used as a negative control for cell toxicity. Error bars indicate mean $\pm \mathrm{SD}(\mathrm{n}=3) . *, P=0.0075 ; * *, P=0.007$.

\section{DISCUSSION AND CONCLUDING REMARKS}

Alternative splicing is quickly gaining momentum as a viable target for molecular therapies [29]. STAT family of proteins is involved in many physiological processes and participates in inflammatory responses and cancer progression. The problem with current approaches of therapeutic targeting of STAT proteins is simultaneous inhibition of opposite functions of STAT isoforms. In this work we are presenting the design of the new approach to overcome this problem.

Splice-switching oligonucleotides are considered a novel class of therapeutics intended to induce therapeutically favorable splice variants of targeted genes [6]. The potential therapeutic benefit of antisense oligonucleotides has been demonstrated by several reports [30-32]. Antisense Morpholino oligonucleotides complementary to exon/intron boundary sequences can block access of the spliceosomal ma- chinery and enhance alternative exon inclusion [23, 33]. The demonstrated approach focused on induction of the specific splice retention that can occur naturally [4]. We have demonstrated that application of Morpholino oligonucleotides to target exon-intron boundary in the pre-mRNA of Stat5B are able to induce intron/exon retention, which was confirmed by semi-quantitative RT-PCR. . While limited delivery efficiency caused incomplete modification of splicing, all targeted Morpholino oligos generated measurable amounts of predicted target products at $559 \mathrm{bp}$ (Figure 2). These results demonstrate that the designed oligonucleotides are capable of enhancing intron retention (Figure 2). Additionally, quantitative RT-PCR analysis confirmed a dose-dependent shift in splicing leading to the specific intron retention (Figure 3 ) and our results indicate that a modified transcript with the additional exon can be efficiently translated into STAT5 $\Delta$ B protein (Figure 4).

Expression levels of STAT5 $\Delta$ B protein after enhancement was still significantly lower than the full 
length form. Although relative protein levels are lower, studies have previously demonstrated that the dominant-negative form has a significantly stronger impact on transcriptional regulation due to a slower rate of deactivation $[4,15]$. Our cell culture data are consistent with previously reported observation regarding the tumor suppressor activity of STAT $5 \Delta \mathrm{B}$ $[10,11]$. Cell proliferation assays revealed that generation of the dominant-negative isoform of STAT5B by splice switching SSOs leads to a dose-dependent decrease in cell proliferation (Figure 5). In addition a clonogenic assay, which reflects the malignant potential of cancer cell lines, indicated a specific and significant reduction in prostate cancer cell viability as induced by SSOs treatment (Figure 6).

These data demonstrate the feasibility of using specific steric-blocking splice-switching oligonucleotides with a complimentary sequence to induce alternative intron/exon retention to convert a proto-oncogene (full length STAT5B) into a tumor suppressor (dominant-negative truncated form of STAT5B) as a possible anti-tumor therapeutic strategy. Moreover, alternative splice-switching oligonucleotides inducing a switch from proto-oncogene to tumor suppressor functions could potentially be used for therapeutic tuning of other STAT proteins.

A common feature of most STATs is alternative splicing, which leads to generation of a dominant-negative isoform. STAT proteins are involved in wide variety of physiological processes including immune response and tumor progression. Ability to modulate their actions and specifically switch function from tumor activating to tumor suppressing would be highly beneficial in many areas of biomedical research. In conclusion we developed and confirmed a novel method to implement steric-blocking splice-switching oligonucleotides for targeted delivery towards the development of novel therapeutic strategies.

\section{AKNOWLEDGEMENTS}

This work was supported by National Institute of Health (SC3GM087201) and by Frances Rusteberg Faculty Fellowship at The University of Texas at Brownsville. The authors thank Dr. Kari Brewer-Savannah and Dr. Daniele Provenzano from UTB for useful suggestions and critical reviewing of the manuscript.

\section{COMPLETING INTERESTS}

The authors declare no competing interests.

\section{REFERENCES}

1. Darnell J.EJr, Kerr I.M, and Stark G.R. Jak-STAT pathways and transcriptional activation in response to IFNs and other extracellular signaling proteins. Science, 1994; 264(5164): 1415-21.

2. Ihle J.N and Kerr I.M. Jaks and Stats in signaling by the cytokine receptor superfamily. Trends Genet, 1995; 11(2): 69-74.

3. Schindler $\mathrm{C}$ and Strehlow I. Cytokines and signaling STAT. Adv Pharmacol, 2000; 47: 113-74.

4. Kazansky A.V, et al. Regulation of mammary gland factor/Stat5a during mammary gland development. Mol Endocrinol, 1995; 9(11): 1598-609.

5. Lim C.P and Cao X . Structure, function, and regulation of STAT proteins. Mol Biosyst, 2006; 2(11): 536-50.

6. Ramos H.L, O'Shea J.J, and Watford W.T. STAT5 isoforms: controversies and clarifications. Biochem J, 2007; 404(1): e1-2.

7. Chen H, et al. VEGF, VEGFRs expressions and activated STATs in ovarian epithelial carcinoma. Gynecol Oncol, 2004; 94(3): 630-5.

8. Debierre-Grockiego F. Anti-apoptotic role of STAT5 in haematopoietic cells and in the pathogenesis of malignancies. Apoptosis, 2004; 9(6): 717-28.

9. $\mathrm{Li} \mathrm{H}$, et al. Activation of signal transducer and activator of transcription 5 in human prostate cancer is associated with high histological grade. Cancer Res, 2004; 64(14): 4774-82.

10. Kazansky A.V and Greenberg N.M. Role STAT5B in Prostate Cancer Progression. (manuscript in preparation), 2005.

11. Kazansky A.V, Spencer D.M, and Greenberg N.M. Activation of signal transducer and activator of transcription 5 is required for progression of autochthonous prostate cancer: evidence from the transgenic adenocarcinoma of the mouse prostate system. Cancer Res, 2003; 63(24): 8757-62.

12. $\mathrm{Yu} \mathrm{H}$ and Jove R. The STATs of cancer--new molecular targets come of age. Nat Rev Cancer, 2004; 4(2): 97-105.

13. Xi S, et al. Constitutive activation of Stat $5 \mathrm{~b}$ contributes to carcinogenesis in vivo. Cancer Res, 2003; 63(20): 6763-71.

14. Morcinek J.C, et al. Activation of STAT5 triggers proliferation and contributes to anti-apoptotic signalling mediated by the oncogenic Xmrk kinase. Oncogene, 2002; 21(11): 1668-78.

15. Kazansky A.V and Rosen J.M. Signal Transducer and Activator of Transcription 5B Potentiates v-Src-mediated Transformation of NIH-3T3 Cells. Cell Growth \& Differentiation, 2001; 12: 1-7.

16. Kazansky A, et al. Differential effects of prolactin and src/abl kinases on the nuclear translocation of STAT5B and STAT5A. The Jornal of Biological Chemistry. 1999; 274(32):22484-92.

17. Dagvadorj A, et al. Transcription factor signal transducer and activator of transcription 5 promotes growth of human prostate cancer cells in vivo. Clin Cancer Res, 2008; 14(5): 1317-24.

18. Fiebig H.H, Maier A, and Burger A.M. Clonogenic assay with established human tumour xenografts: correlation of in vitro to in vivo activity as a basis for anticancer drug discovery. Eur J Cancer, 2004;40(6): 802-20.

19. $\mathrm{Gu} \mathrm{L}$, et al. Stat 5 promotes metastatic behavior of human prostate cancer cells in vitro and in vivo. Endocr Relat Cancer, 2010; 17(2): 481-93.

20. Santos C.I and Costa-Pereira A.P. Signal transducers and activators of transcription-from cytokine signalling to cancer biology. Biochim Biophys Acta, 2011; 1816(1): 38-49.

21. Bauman J, Jearawiriyapaisarn N, and Kole R. Therapeutic potential of splice-switching oligonucleotides. Oligonucleotides, 2009. 19(1): 1-13.

22. Singh N.K, et al. Splicing of a critical exon of human Survival Motor Neuron is regulated by a unique silencer element located in the last intron. Mol Cell Biol, 2006; 26(4): 1333-46.

23. Hua $Y$, et al. Enhancement of SMN2 exon 7 inclusion by antisense oligonucleotides targeting the exon. PLoS Biol, 2007; 5(4): e73.

24. Sazani P and Kole R. Therapeutic potential of antisense oligonucleotides as modulators of alternative splicing. J Clin Invest, 2003; 112(4): 481-6.

25. Sazani $P$, et al. Nuclear antisense effects of neutral, anionic and cationic oligonucleotide analogs. Nucleic Acids Res, 2001;29(19): 3965-74.

26. Roberts J, et al. Efficient and persistent splice switching by systemically delivered LNA oligonucleotides in mice. Mol Ther, 2006; 14(4): 471-5.

27. Pfaffl M.W. A new mathematical model for relative quantification in real-time RT-PCR. Nucleic Acids Res, 2001; 29(9): e45.

28. Cao $X$, et al. Activation and association of Stat3 with Src in v-Src-transformed cell lines. Mol Cell Biol, 1996; 16(4): 1595-603.

29. Bauman J.A, et al. Anti-tumor activity of splice-switching oligonucleotides. Nucleic Acids Res, 2010;38(22): 8348-56.

30. Montgomery R.L, et al. Therapeutic inhibition of miR-208a improves cardiac function and survival during heart failure. Circulation. 2011;124(14): 1537-47. 
31. Muntoni F, and Wood M.J. Targeting RNA to treat neuromuscular disease. Nat Rev Drug Discov. 2011;10(8): 621-37.

32. Feng J, et al. Antisense oligodeoxynucleotides targeting ATM strengthen apoptosis of laryngeal squamous cell carcinoma grown in nude mice. J Exp Clin Cancer Res. 2011;30: 43.

33. Singh N.N, et al. A short antisense oligonucleotide masking a unique intronic motif prevents skipping of a critical exon in spinal muscular atrophy. RNA Biol, 2009; 6(3): 341-50. 